\title{
ACADÊMICOS DE ENFERMAGEM E SEU AUTOCUIDADO EM RELAÇÃO A DOENÇAS SEXUALMENTE TRANSMISSÍVEIS
}

\section{NURSING STUDENTS AND THEIR SELF-CARE IN RELATION TO SEXUALLY TRANSMITTED DISEASES}

\section{ESTUDIANTES DE ENFERMERÍA Y SU AUTOCUIDADO EN RELACIÓN CON ENFERMEDADES DE TRANSMISIÓN SEXUAL}

\author{
Amina Regina Silva ${ }^{1}$, Maria Itayra Padilha ${ }^{2}$
}

\section{RESUMO}

Objetivo: conhecer as medidas de autocuidado utilizadas por estudantes de graduação em enfermagem da Universidade Federal de Santa Catarina em relação a doenças sexualmente transmissíveis. Método: trata-se de uma pesquisa quantitativa descritiva, para cuja realização foi aplicado um questionário direcionado a alunos de todas as fases do curso de graduação em enfermagem, totalizando uma amostra de 149 estudan tes. Resultados/discussão: os dados obtidos por meio dessa metodologia revelaram divergências entre o conhecimento e a ação, levando-nos a refletir que, apesar do acesso à informação, os estudantes frequentemente negligenciam a importância do autocuidado no âmbito da vida pessoal. Conclusão: a divergência entre ação e conhecimento justifica relacionar o presente estudo com as referências ao déficit do autocuidado assinaladas na teoria do autocuidado de Dorothea Orem, por cujo efeito os sujeitos do estudo não conseguem, individualmente, exercer o autocuidado de maneira eficaz e autossuficiente.

Descritores: HIV; Doenças Sexualmente Transmissíveis; Autocuidado; Estudantes de Enfermagem.

\section{ABSTRACT}

Objective: to know the self-care measures used by undergraduate students in nursing at the Federal University of Santa Catarina in relation to sexually transmitted diseases. Method: it is a descriptive quantitative research, for whose achievement was a questionnaire directed to students of all stages of the undergraduate degree in nursing, a total sample of 149 students. Results / discussion: the data obtained through this methodology revealed differences between

\footnotetext{
${ }^{1}$ Enfermeira e mestranda pela Universidade Federal de Santa Catarina

${ }^{2}$ Pós-doutorado pela Universidade de Toronto e docente na Universidade Federal de Santa Catarina.
} 
knowledge and action, leading us to think that despite access to information, students often neglect the importance of self-care in the context of personal life. Conclusion: the difference between action and knowledge justifies relate this study with references to the deficit of selfcare marked on the theory of Dorothea Orem, for which purpose the study subjects cannot individually exercise self-care effective and self-sufficient way.

Keywords: HIV, Sexually Transmitted Diseases, Self Care, Students Nursing.

\section{RESUMEN}

Objetivo: conocer las medidas de cuidados personales utilizados por los estudiantes de grado en enfermería de la Universidad Federal de Santa Catarina, en relación con las enfermedades de transmisión sexual. Método: es una investigación cuantitativa descriptiva, al que ha aplicado un cuestionario dirigido a los estudiantes de todas las etapas de la licenciatura en enfermería, una muestra total de 149 estudiantes. Resultados/Discusión: los datos obtenidos con esta metodología revelaron diferencias entre el conocimiento y la acción, que nos lleva a pensar que a pesar del acceso a la información, los estudiantes con frecuencia descuidan la importancia de los autocuidados en el contexto de la vida personal. Conclusión: la diferencia entre la acción y el conocimiento justifica este estudio se relacionan con referencias al déficit de autocuidado marcado en la teoría de Dorothea Orem, por el efecto los sujetos del estudio no pueden ejercer individualmente autocuidado manera eficaz y autosuficiente.

Descriptores: VIH; Enfermedades de Transmisión Sexual; Autocuidado; Estudiantes de Enfermería.

\section{INTRODUÇÃO}

A Síndrome da Imunodeficiência Adquirida (Aids) é uma doença que, desde o seu surgimento até a atualidade, tem gerado intensas polêmicas no contexto de saúde pública mundial e já foi considerada a pior epidemia do século XX. Essa percepção pode ser relacionada ao fato de a Aids ser uma doença que causa sério comprometimento ao sistema imunológico das pessoas infectadas pelo Vírus da Imunodeficiência Humana (HIV), além de não ter ainda cura efetiva, apenas tratamento a ser aplicado, mesmo com todos os avanços atuais. ${ }^{1}$

A Aids é caracterizada como uma Doença Sexualmente Transmissível (DST), uma vez que a via mais comum de contaminação é através de relações sexuais 
sem o uso adequado de preservativos; contudo, existem outras de formas de adquirir a doença, tais como contato direto com sangue e fluidos de indivíduos contaminados com HIV. ${ }^{2}$ Essas características, combinadas com a ocorrência da epidemia de Aids, despertaram o interesse coletivo acerca de como exercemos nossa sexualidade.

Além disso, as DST frequentemente são apontadas como um fator de risco adicional, pois deixam o organismo mais vulnerável a fatores exógenos e, consequentemente, acabam sendo um facilitador no processo de transmissão do HIV. Isso significa que o indivíduo já possuidor de alguma das doenças classificadas como DST está mais vulnerável ao HIV do que os demais. ${ }^{2}$

Por ser a Aids uma doença atualmente considerada incurável, já se sabe que a maneira mais efetiva de combater a Aids e DST de maneira geral é a prevenção. Por isso, o aconselhamento, na forma de uma prevenção individual, surgiu como uma estratégia elaborada pela equipe nacional do Ministério da Saúde, com a finalidade de trabalhar com a identificação do risco e propiciar uma reflexão sobre medidas preventivas junto ao indivíduo. ${ }^{3-4}$
Dentre os profissionais da área da saúde, destacamos aqui os que atuam na área da enfermagem, na medida em que tais profissionais, de acordo com a sistemática de cuidados da enfermagem, têm grande contato e vínculo com o cliente em todos os níveis de atenção, além da aptidão e do conhecimento necessários para realizar essa tarefa. ${ }^{5}$

Quando se fala em prevenção de DST de maneira geral, a população jovem sobressai como um grupo importante a ser considerado. Cabe ressaltar, inclusive, que a população jovem é definida como prioridade nas campanhas de prevenção promovidas pela Organização das Nações Unidas (ONU). Tal fato pode ser relacionado, em parte, ao reconhecimento da vulnerabilidade desse grupo, destacando-se aqui, como fatores predisponentes, a sensação de onipotência, falta de informações adequadas, profissionais despreparados para lidar com adolescentes e baixa qualidade dos serviços de saúde, dentre outros. ${ }^{6}$

Estudos revelam que boa parte da população universitária da área de saúde, em especial do curso de graduação em enfermagem, não demonstra efetiva preocupação com a aquisição de doenças sexualmente transmissíveis; esse fato, porém, não está necessariamente 
relacionado com a falta de conhecimentos sobre tais doenças. ${ }^{7}$

Destacamos, ainda, a importante ligação entre a enfermagem e o autocuidado, tendo em vista que o cuidado é considerado o objeto epistemológico da enfermagem, o que torna, consequentemente, as atividades de educação relacionadas ao autocuidado uma das competências da profissão. As definições de autocuidado são muito variáveis entre as culturas. De forma geral, o autocuidado é uma função reguladora, por meio da qual os indivíduos adotam atitudes e comportamentos que visam beneficiar sua saúde, nos requisitos vitais, no desenvolvimento e funcionamento de forma integral, sem supervisão médica formal. $^{8}$

Destacamos aqui a importância do enfermeiro no papel de prevenção de DST; no entanto, para que esse trabalho seja efetuado de maneira correta e eficiente, os profissionais devem ser capacitados continuamente para tal atividade. Nesse sentido, o preparo deve ser iniciado na formação acadêmica, pois os conhecimentos profissionais, na grande maioria das vezes, são adquiridos durante a permanência do indivíduo no curso de graduação. Enfatizamos a importância de avaliar se o conhecimento adquirido no curso de graduação em enfermagem das populações universitárias, cuja faixa etária predominante se enquadra naquela considerada vulnerável pela ONU, está sendo aplicado na vida pessoal desses estudantes. Tal avaliação visa identificar se os futuros profissionais da área da saúde estão de fato preparados para exercer a árdua atividade, e se já conhecem e utilizam tais princípios de prevenção, diagnóstico e tratamento de HIV/Aids e DST no âmbito da vida pessoal.

Esses fatos nos fazem refletir sobre o real conhecimento desses jovens acerca da temática e, com base no nível de percepção que demonstram, como é feito de fato o autocuidado para a prevenção dessas doenças. A questão que impulsiona este estudo é: como é realizado o autocuidado em relação ao HIV/Aids e DST na vida pessoal de estudantes do curso de graduação em enfermagem da Universidade Federal de Santa Catarina (UFSC)?

Portanto, esse estudo tem por objetivo conhecer as medidas de autocuidado utilizadas por estudantes de graduação em enfermagem da UFSC em relação a doenças sexualmente transmissíveis no âmbito da vida pessoal.

A fim de estabelecer uma melhor compreensão da contextualização desta 
pesquisa, foi escolhida a Teoria do Autocuidado de Dorothea Orem como referencial teórico, pois é a que melhor reflete a temática em questão neste estudo. A Teoria de Dorothea Orem tem sido muito utilizada na esfera das questões atuais na prática de enfermagem, não apenas no que se refere especificamente a determinadas patologias, mas porque diz respeito a uma ampla gama de dimensões do cuidado, provando que a teoria tem uma grande aplicabilidade.

$\mathrm{Na}$ dimensão da teoria do autocuidado, Dorothea Orem postula que, quando um indivíduo não tem condições de suprir suas próprias necessidades básicas, a enfermagem entra como um sistema de ajuda complementar para o autocuidado. ${ }^{9}$

Ainda em conformidade com os fatores anteriormente expostos, destacamos aqui que a Teoria do Autocuidado de Dorothéa Orem contempla, em sua dimensão geral, três teorias interrelacionadas, com as quais trabalhamos aqui na busca por embasamento em um conceituado e tradicional autor nesta temática: ${ }^{10}$

-Teoria do auto cuidado: "Autocuidado é a prática de atividades, iniciadas e executadas pelo indivíduo, em seu próprio benefício, para manutenção da vida, da saúde e do bem-estar". ${ }^{10}$

-Teoria do déficit de autocuidado: essa teoria constitui a essência da teoria geral de enfermagem de Orem, uma vez que estabelece o momento em que há necessidade de intervenção da enfermagem. Assim, a enfermagem passa a ser necessária quando um adulto (ou aquele que tem a guarda) encontra-se incapacitado ou limitado para prover autocuidado contínuo e eficaz. ${ }^{10}$

- Teoria de sistemas de enfermagem: esse sistema planejado pelo profissional se baseia nas necessidades de autocuidado e nas capacidades do paciente para a execução das atividades de autocuidado. ${ }^{10}$

\section{MÉTODO}

Esta pesquisa compõe-se de um estudo com abordagem quantitativa descritiva, em que o respectivo instrumento de coleta de dados continha também características qualitativas, conforme será detalhado posteriormente.

A população do estudo foi inicialmente constituída pela totalidade de alunos regularmente matriculados no curso de graduação em enfermagem da UFSC, independentemente de fase, currículo ou número de disciplinas que o aluno estivesse cursando durante o período de coleta de dados. 
De acordo com o sistema da UFSC, no período de coleta de dados o curso de graduação em enfermagem possuía 292 estudantes regularmente matriculados. Porém, mediante uma averiguação com os respectivos professores das disciplinas, o número de estudantes que frequentava regularmente as aulas foi determinado em 255. Além disso, na realização desta pesquisa conseguimos contemplar 149 estudantes como amostra estudada, ou seja, $58,43 \%$ de toda a amostra inicial.

Vale notar, ainda, que foi realizado um teste com instrumento piloto, aplicado com 10 alunos do curso de graduação, para avaliar a qualidade do instrumento de coleta de dados. De acordo com o relatado pelos estudantes, as alterações necessárias para melhorar o instrumento foram concretizadas.

Para que a avaliação dos alunos em relação ao autocuidado e conhecimento em HIV/Aids e DST fosse realizada de maneira a gerar os resultados mais fidedignos possíveis, foi elaborado um questionário com 28 questões, que visam conhecer o perfil do aluno e definir quais medidas de autocuidado são utilizadas por eles. Dessas questões, o instrumento contou com 27 de abordagem quantitativa e uma única questão com abordagem qualitativa, buscando assim identificar qual o motivo específico para o estudante ter ou não medo de adquirir alguma DST.

A coleta de dados nas turmas da $1^{\text {a }}$ à $6^{\mathrm{a}}$ fase, previamente agendada com os professores, foi realizada no campus da UFSC durante os horários de aula. Já na $7^{\mathrm{a}}$ e $8^{\mathrm{a}}$ fases do curso, os períodos finais, em razão do que os estudantes ficam distribuídos nos campos de prática, não foi possível realizar a coleta pessoalmente, motivo pelo qual o instrumento foi aplicado via e-mail. O período de coleta de dados foi compreendido entre os meses de fevereiro e março de 2014. Após a coleta de dados, estes foram organizados e categorizados por semelhanças de informações e apresentados em forma de tabela com frequência simples.

Corroborando e respeitando o préestabelecido nas normas, regras e diretrizes propostas pelo Comitê de pesquisas envolvendo seres humanos, definidas na Resolução 466/12 do Conselho Nacional de Saúde - Ministério da Saúde, esta pesquisa foi submetida e aprovada pelo Comitê de Ética em Pesquisa com Seres Humanos da UFSC, sob o número de parecer 526.350.

\section{RESULTADOS}

Após a coleta de dados, os resultados oriundos dos 149 questionários respondidos foram individualmente 
computados e analisados. Iniciaremos a apresentação dos resultados pelo perfil dos sujeitos nos aspectos relativos à vida pessoal dos estudantes. Podemos traçar, como perfil predominante, heterossexuais (94,63\%), namorando (51\%), com uma média de relacionamento superior a dois anos de duração e sem filhos $(92,61 \%)$.

A respeito da vida sexual dos estudantes, quando questionados sobre a idade média da primeira relação sexual, tivemos, em ordem decrescente, de 15 a 17 anos (51\%), de 18 a 20 anos (22,81\%), não iniciaram atividade sexual (10,73\%), de 12 a 14 anos de idade $(10,73 \%)$ e por último os que tiveram a primeira relação sexual com mais de 20 anos de idade (4,69\%).

No que se refere à média de parceiros sexuais durante a vida, obtivemos $10,73 \%$ que não iniciaram atividade sexual, 5,36\% que tiveram de 1 a 3 parceiros sexuais; $22,81 \%$ que tiveram de 4 a 9 parceiros, 9,39\% que tiveram de 10 a 14 parceiros, $2,01 \%$ que tiveram de 15 a 19 parceiros e $1,34 \%$ tiveram mais de 20 parceiros sexuais.

Quando questionados sobre a utilização de métodos contraceptivos e medidas de prevenção contra as doenças sexualmente transmissíveis, $73,15 \%$ dos alunos sempre consideram importante o uso de preservativos nas relações sexuais,
19,46\% quase sempre consideram, 6,04\% às vezes consideram e 1,34\% quase nunca consideram, destacando ainda que nenhum aluno respondeu que a utilização de preservativos nunca é importante. Porém, quando questionados se usam preservativos nas relações sexuais, obtivemos uma informação diferente da anterior: $\quad 35,57 \%$ dos estudantes declararam usar sempre preservativos, $23,48 \%$ quase sempre, $14,76 \%$ às vezes, $10,06 \%$ quase nunca, $10,73 \%$ nunca e $5,36 \%$ optaram por não responder ao questionamento. Apresentamos aqui a tabela 1, que demonstra os motivos que levam os estudantes a não usar preservativos, e a tabela 2 , que demonstra os métodos contraceptivos mais utilizados por esses estudantes.

Tabela 1 - Principais motivos para os estudantes não utilizarem preservativos em todas as relações sexuais, Florianópolis, 2014.

\begin{tabular}{ll}
\hline Motivo & Percentual \\
\hline Confiança no parceiro(a) & $79,10 \%$ \\
Outro motivo & $10,44 \%$ \\
Momento do prazer "fala & $4,47 \%$ \\
mais alto" & \\
Esquecimento & $2,98 \%$ \\
Sentimento & $2,98 \%$ \\
invulnerabilidade & \\
\hline
\end{tabular}

ISSN 2317-1154 
Curiosidade

Por exposição hospitalar a $\quad 14,28 \%$

Tabela 2 - Os métodos contraceptivos e preventivos mais utilizados dentre os estudantes, Florianópolis, 2014

\begin{tabular}{|c|c|}
\hline Método & Percentual \\
\hline Anticoncepcionais, de uso da & $61,83 \%$ \\
\hline parceiro & \\
\hline injetáveis & \\
\hline adesivos) & \\
\hline Preservativos (feminino e & $30,53 \%$ \\
\hline masculino) & \\
\hline Nenhum & $5,34 \%$ \\
\hline Dispositivo intrauterino & $1,52 \%$ \\
\hline Métodos naturais (tabelinha, & $0,76 \%$ \\
\hline muco ou billing, coito & \\
\hline interrompido) & \\
\hline
\end{tabular}

Quando questionados sobre a realização de testes e exames de detecção de HIV/Aids e DST, 56,37\% declararam já ter realizado algum exame do gênero e $43,62 \%$ nunca realizaram tais testes e exames. E os motivos pelos quais tais alunos realizaram estes testes, estão demonstrados a seguir na tabela 3 .

Tabela 3 - Motivos pelos quais os alunos realizaram o teste de deteção de HIV ou DST, Florianópolis, 2014

\begin{tabular}{l}
\hline Motivo do exame \\
\hline Para doação de sangue ou $35,71 \%$ \\
hemoderivados \\
Rev Enferm Atenção Saúde [Online]. Jan/Jul 2016; 5(1):36-50
\end{tabular}

fluidos corpóreos

Por comportamentos de 11,90

riscos

Para exames de rotina $5,95 \%$

durante o pré-natal

Para detecção de outros meios de cuidados de prevenção relativa ao HIV/Aids e DST, os estudantes foram questionados quanto ao uso de drogas injetáveis, e $100 \%$ dos estudantes negaram o uso de algum tipo de drogas injetáveis. Quanto à influência do curso de graduação em enfermagem na mudança da percepção e conhecimento dos estudantes com relação ao HIV/Aids e DST, os estudantes se manifestaram do seguinte modo: $24,16 \%$ declararam que sua percepção mudou completamente, $38,92 \%$ que a sua percepção mudou parcialmente e 30,20\% declararam que não houve mudanças na sua percepção. Um fato interessante sobre a percepção da mudança de conhecimentos referentes ao ensino teórico prático da temática HIV/Aids e DST é que 42,22\% dos que declararam não ter havido mudança no seu nível de conhecimentos após a entrada no curso são da primeira fase, o que em parte justifica tal resultado. 
Finalizando o instrumento, utilizamos uma questão com abordagem qualitativa, visando obter respostas cujas justificativas fossem mais específicas. Assim, quando os estudantes foram questionados se tinham receio ou medo de adquirir HIV/Aids ou DST, 83,89\% responderam afirmativamente e os outros $16,10 \%$ negaram tal fato.

Justificando o receio ou medo de adquirir HIV/Aids ou DST, apontamos aqui algumas das respostas dos estudantes:

Adquirir esta doença é correr risco de diminuir minha qualidade e expectativa de vida (aluno da $1^{\mathrm{a}}$ fase).

Por mais que eu não apresente comportamento de risco, pois meu parceiro e eu realizamos periodicamente exames e não mantivemos relações sexuais com outras pessoas sem uso de preservativos, há sempre o receio de contrair a doença, através, por exemplo, (mas este só se explica na minha situação como futuro profissional de enfermagem) do manuseio de perfurocortantes infectados (aluno da $2^{\mathrm{a}}$ fase).

É uma doença que terás que tratar pelo resto da vida, que não será nunca mais a mesma. Mesmo com o tratamento, vejo o medo da morte em pessoas próximas a mim que possuem a doença (aluno $3^{\mathrm{a}}$ fase).

Devido à rotina hospitalar e da área da saúde, estamos expostos a riscos todos os dias (aluno da $4^{\mathrm{a}}$ fase).

Preconceitos, e a própria situação da doença por sua evolução e complicações, muitas vezes convivendo com a morte (aluno da $4^{\mathrm{a}}$ fase).

Depois de fazer estágio no centro de testagem $e$ aconselhamento do vírus HIV percebi que a Aids não é algo que vem estampado na cara das pessoas. Fiquei com medo de ver quantas pessoas possuem HIV. Depois disso fiquei com medo de fazer o exame, pois eu sei com quem transei, mas não sei com quem a pessoa que transei transou. Depois de um tempo fiz todos os exames DST e HIV e deu tudo não reagente (aluno da $5^{\mathrm{a}}$ fase).

Exposição a acidentes devido à formação profissional. Comportamento de risco é uma possibilidade, principalmente quando há consumo de álcool associado (aluno da $6^{\mathrm{a}}$ fase).

Medo, pois é uma doença difícil de lidar, principalmente pelo preconceito. Eu teria que me tratar pelo resto da vida, é complicado, possuo receio relacionado à exposição a que estamos susceptiveis no ambiente de trabalho (aluno da $7^{\mathrm{a}}$ fase).

Entre os que responderam não ter receio ou medo de adquirir HIV/Aids ou DST, alguns estudantes justificaram com as seguintes respostas:

Porque tomo medidas de prevenção (aluno da $1^{\mathrm{a}}$ fase).

Tenho um único parceiro na minha vida, de extrema confiança. Não tive e nunca vou ter 
relação sexual com mais ninguém (aluno da $1^{\mathrm{a}}$ fase).

Não me considero dentro de nenhum grupo de risco (aluno da $2^{\mathrm{a}}$ fase).

Sempre utilizo preservativo, mesmo em caso de relacionamento sério ou união estável. Todas as vezes. Nunca deixei de usar, nunca tive relação sexual sem camisinha. Já utilizei camisinha feminina pela falta de preservativo masculino (aluno da $6^{\mathrm{a}}$ fase).

Acredito que eu tenho informação suficiente $e$ recursos para me cuidar e me prevenir (aluno da $6^{\text {a }}$ fase).

Os avanços científicos e a sofisticada atenção prestada aos portadores de HIV/Aids e DST atualmente fazem com que os indivíduos tenham expressiva qualidade de vida, caso tenham adesão ao tratamento (aluno da $7^{\mathrm{a}}$ fase).

\section{DISCUSSÃO}

Como já exposto anteriormente, o objetivo deste estudo foi identificar como é realizado o autocuidado pelos estudantes do curso de enfermagem da UFSC em relação ao HIV/Aids e DST, no âmbito da vida pessoal.

Conforme a análise de dados, a idade média desses estudantes é entre 18 e 22 anos, a qual está dentro de uma das principais faixas etárias atingidas por essas doenças, que é a de 15 a 29 anos, conforme os dados contidos no Boletim
Epidemiológico Aids e DST do Ministério da Saúde do ano de 2015. ${ }^{11}$ Tais dados reforçam a importância desta pesquisa, qual seja, estudar esse grupo de acadêmicos incluído no principal grupo de risco sujeito à incidência dessas doenças no Brasil.

Quanto ao perfil sexual dos estudantes, o fato de a maioria dos estudantes já ter vivenciado relações sexuais é bastante semelhante ao resultado de outros estudos que trabalharam com temáticas dessa mesma linha. Existem autores que fizeram essa mesma afirmação com referência às relações sexuais, embora a média de idade dos estudantes incluídos em tais pesquisas seja um pouco inferior à dos incluídos no presente estudo. ${ }^{12}$

Quando questionados se acham importante a utilização de medidas de prevenção (preservativos) contra as doenças sexualmente transmissíveis nas relações sexuais, a maioria dos estudantes sempre considerou importante o uso de preservativos nas relações sexuais. Essa é uma característica já reafirmada em outros estudos, os quais resultaram em que a quase totalidade dos estudantes universitários participantes da pesquisa consideraram que os adolescentes deveriam usar preservativos em todas as relações sexuais. $^{13}$ 
Posteriormente, os estudantes da pesquisa foram questionados se usam preservativos nas relações sexuais - apenas uma minoria respondeu sempre usar preservativos. As justificativas dadas pelos estudantes que afirmaram não fazer o uso de preservativos foram, na grande maioria, a confiança no parceiro, enquanto alguns citaram que o momento do prazer "fala mais alto" e outros motivos em menor número e relevância.

Os dados obtidos nesta pesquisa foram analisados utilizando-se como base o referencial da teoria do autocuidado, conforme já exposto anteriormente. Nessa abordagem, procuramos entender a relação direta da forma pela qual os estudantes avaliados exercem o autocuidado na vida pessoal, levando em consideração os conceitos de autocuidado de Orem. ${ }^{10} \mathrm{~A}$ análise dos resultados expôs um evidente desequilíbrio, na medida em que os cuidados incorporados no cotidiano dos alunos são inversamente proporcionais aos conhecimentos adquiridos em sala de aula e aos conhecimentos pré-existentes.

De fato, ainda que os estudantes tenham conhecimento da importância do autocuidado para a manutenção da sua saúde e bem estar, na grande maioria das vezes esses fatores não são levados em conta na prática cotidiana, o que é possível visualizar por meio dos dados já mencionados nesta pesquisa.

Face ao exposto, é razoável concluir, nessa relação desproporcional em que a capacidade de autocuidado é inferior à demanda, que o déficit de autocuidado inabilita a realização de um autocuidado eficaz quando se trata de prevenção de DST.

Desse modo, os resultados desta pesquisa permitem inserir os estudantes na vertente do déficit do autocuidado presente na teoria de autocuidado de Orem, dado que eles precisariam de auxílio complementar para conseguir promover seu autocuidado de maneira eficiente. Nesse caso, podemos admitir como opção um auxílio por meio de medidas educativas - e não técnicas, uma vez que não existe a limitação de ordem física e sim psicológica, que pode estar relacionada a várias origens. ${ }^{9-10}$

Quanto aos métodos contraceptivos utilizados pelos indivíduos desta pesquisa, a maioria dos estudantes citou o uso de anticoncepcionais como principal método. Tal resultado diverge de grande parte dos estudos já realizados na área com temas e públicos bem semelhantes, já que, nos demais estudos, a maior parte do grupo estudado cita o preservativo como o método de primeira escolha. ${ }^{14}$ 
No que diz respeito ao comportamento sexual de risco, observa-se que a maioria desses estudantes se submeteu a práticas sexuais de risco, sem o uso de preservativos, dado este que nos remete a estudos que indicam, de maneira geral, como é notável que os estudantes da área da saúde, em especial do curso de enfermagem, não demonstrem efetiva preocupação com a aquisição de doenças sexualmente transmissíveis. Conforme os dados levantados, esse fato não está necessariamente relacionado com a falta de conhecimento sobre DST, reafirmando que conhecimento e ação não estão diretamente ligados. ${ }^{7}$ Corroborando com essa justificativa, temos estudos ainda que trazem, como explicação plausível para a exposição dos jovens a comportamentos sexuais que ponham em risco sua saúde, uma possível predisposição comum biológica ou sociocultural. Porém, ainda consideramos essas justificativas como suposições, tendo em vista que ainda não existem estudos científicos comprovando suas teorias. $^{12}$

É possível ponderar que a prática contraceptiva de primeira escolha destes estudantes, o anticoncepcional, está intimamente relacionada com uma preocupação primordial na visão dos jovens, qual seja, os resultados dão suporte à conclusão de que, para esses jovens, a prevenção de gravidez é mais importante do que a prevenção de DST, como é o caso do HIV/Aids. Isto pode ser justificado pelos motivos já mencionados, referentes ao não uso de preservativos.

Para finalizar os pontos que devem ser considerados para discussão, temos o levantamento de dados feito quando os alunos foram questionados sobre ter ou não receio e medo de adquirir o HIV/Aids e DST de modo geral. Verificamos que a grande maioria afirmou ter medo de adquirir doenças e apenas uma minoria negou tal fato. Dentre as justificativas dos estudantes que afirmaram ter medo ou receio, os motivos mais citados foram a diminuição da qualidade e expectativa de vida e o preconceito. Desde o surgimento do HIV/Aids até a atualidade, essa doença sempre foi cercada de preconceito, e boa parte disso se deve à constante associação da doença com pessoas homossexuais, trabalhadores do sexo e usuários de drogas, e à caracterização da Aids como uma doença severa e fatal. ${ }^{1}$

Temos um estudo que traz algumas justificativas para essas questões, afirmando que isso se deve, em parte, ao fato de que a Aids é uma doença que ainda não tem cura e seu principal meio de transmissão é por via sexual, o que dá 
margem a muitos pré-julgamentos e preconceitos de gênero, tornando-a uma doença difícil de ser encarada. ${ }^{15}$ Outra justificativa bastante abordada foi a impossibilidade de identificar quem é ou não portador do vírus HIV, já que o estereótipo alusivo ao paciente portador do HIV mudou ao longo do tempo, pois, enquanto nos primórdios da doença girava em torno da aparência física de debilidade extrema, posteriormente foi desconstruído pela melhoria no tratamento destes indivíduos. $^{16}$

As justificativas dos estudantes para a ausência de receio de contrair DST nos remetem novamente a Orem e sua teoria, tornando mais evidente a incompreensão desses estudantes acerca do autocuidado. Com base em tais afirmativas, partimos do princípio de que todos os sujeitos possuem potencial para realizar o autocuidado; porém, algumas vezes é necessária a oferta de subsídios para contemplar esse cuidado. Isso reafirma a aplicabilidade da teoria do déficit de autocuidado de Orem a esse grupo, devido à evidente desproporção entre informações, conhecimentos e ações dos jovens estudados nesta pesquisa.

Sabemos que a graduação é responsável por fornecer fomentos e subsídios teóricos a tais estudantes; entretanto, a complexidade do assunto impossibilita a abrangência total desta problemática apenas no espaço de tempo disponível no curso de graduação. Os estudantes aqui pesquisados apresentaram diversas contradições e fragilidades a respeito do exercício do autocuidado, o que contribuiu com a reflexão acerca de sua formação e as futuras dificuldades a serem enfrentadas, tanto no que diz respeito ao próprio autocuidado quanto no que diz respeito ao autocuidado de sua futura clientela.

\section{CONCLUSÃO}

Com relação aos resultados obtidos na presente pesquisa, destacamos aqui o modo com que os estudantes de enfermagem estão exercendo o seu autocuidado, visivelmente realizado de maneira ineficaz, principalmente no que se refere ao uso de preservativos em relações sexuais.

Esse achado nos remete ao autocuidado da amostra estudada, nos fazendo embasar tais resultados no modelo teórico de Orem, que diz respeito ao déficit de autocuidado, por cujo efeito os indivíduos não são capazes, por si próprios, de realizar suas ações de autocuidado, demandando auxílio para tal. Por auxílio, entendemos a adoção de ações 
teóricas a fim de conscientizar tais estudantes acerca da importância do autocuidado no âmbito da vida pessoal e principalmente sexual, realizado de maneira correta, já que tais estudantes não apresentam deficiências físicas propriamente ditas que impeçam seu autocuidado. Tais aspectos ganham maior relevância quando lembramos que esses estudantes, no futuro, serão os enfermeiros com responsabilidades por ações de promoção no âmbito de saúde pública e coletiva, incluindo ações de conscientização da população acerca do autocuidado.

Por fim, salientamos, como limitação do presente estudo, o fato de não ter sido possível abordar a totalidade dos estudantes de enfermagem na universidade pesquisada, o que inviabilizou reproduzir a realidade total da população pesquisada.

\section{REFERÊNCIAS}

1. Bastiani JAN. Trajetória histórica da AIDS em Florianópolis (1986 - 2006) [dissertação]. Florianópolis (SC): Universidade Fedeeral de Santa Catarina, Pós Graduação em Enfermagem, Universidade Federal de Santa Catarina, 2010.

2. Reis $\mathrm{CB}$, Bernardes EB. What happens behind bars: prevention strategies developed in civilian police stations against HIV/AIDS and other sexually transmitted diseases. Ciênc saúde coletiva [Internet]. 2011 [Citado em 29 Abr 2014]; 16(7):3331-8. Disponível em: http://www.scielo.br/pdf/csc/v16n7/32.pdf 3. Pequeno CS, Macedo SM, Miranda KCL. Aconselhamento em HIV/AIDS: pressupostos teóricos para uma prática clínica fundamentada. Rev bras enferm [Internet]. 2013 [Citado em 29 Abr 2014]; 66(3):437-41. Disponível em: http://www.scielo.br/scielo.php?script=sci_ arttext\&pid=S0034-

$71672013000300020 \& \operatorname{lng}=\mathrm{en} \& \mathrm{nrm}=\mathrm{iso} \& \mathrm{t}$ $\operatorname{lng}=\mathrm{pt}$

4. Praça NS, Freitas P, Kimura AF. Knowledge about HIV/AIDS on women's health: a descriptive study with undergraduates. Online Braz J Nurs [Internet] 2013 [Cited 2014 Abr 29]; 12(2):359-76. Available from: http://www.objnursing.uff.br/index.php/nur sing/article/view/3831/pdf_2

5. Vieira AC, Silveira LC, Franco TB. A formação clínica e a produção do cuidado em saúde e na enfermagem. Trab educ saúde [Internet]. 2011 [Citado em Abr 19 2014]; 9(1):9-24. Disponível em: http://www.scielo.br/scielo.php?script=sci _arttext\&pid=S19.

6. Val LF, Silva JAS, Rincón LA, Lina RHA, Barbosa RL, Nichiata LYI. Estudantes do ensino medio e o conhecimento em HIV/AIDS: que mudou em dez anos?. Rev esc enferm USP [Internet]. 2013 [Citado em 19 Abr 2014]; 47(3):702-8. Disponível em:http://www.scielo.br/scielo.php?script= sci_arttext\&pid=S0080-

62342013000300702.

7. Santos I, Rocha RPF, Berardinelli LMM. Needs of nursing guidance for selfcare of clients on hemodialysis therapy. Rev bra enferm [Internet]. 2011 [Cited 2014 Abr 29]; 64(2):335-42. Available from:http://www.scielo.br/scielo.php?scrip $\mathrm{t}=$ sci_arttext\&pid=S0034-

71672011000200018

8. Baquedano IR, Santos MA, Teixeira CRS, Martins TA, Zanetti ML. Factors related to self-care in diabetes mellitus patients attended at na emergency service 
in Mexico. Rev esc enferm USP [Internet]. 2010 Dez [Cited 2014 Abr 29]; 44(4):1013-9. Available from: http://www.scielo.br/scielo.php?script=sci _arttext\&pid=S008062342010000400023\&lng=pt\&nrm=iso 9. Nogueira M, Silva M, Mata A. A teoria do autocuidado e sua aplicabilidade para a enfermagem no programa saúde da família. Covibra [Internet]. 2013. Disponível em: http://www.convibra.com.br/upload/paper/ 2012/70/2012_70_4028.pdf

10. Foster PC, Janssens NP, Dorothe EO. In: George JB. Teorias de enfermagem: os fundamentos para a prática profissional. Artes médicas. 1993. P. 90-108.

11. Ministério da Saúde (Brasil), Secretaria de Atenção à Saúde. Boletim Epidemiológico de Aids e DST: Ministério da Saúde; 2015.

12. Campo-Arias A, Ceballo GA, Herazo E. Prevalence of pattern of risky behaviors for reproductive and sexual health among middle- and high-school students. Rev Latino-am enfermagem [Internet]. 2010 [Cited 2014 Abr 29]. 18(2):170-4. Available from: http://www.scielo.br/scielo.php?script=sci _arttext\&pid=S0080-62342013000300702 13. Rebello LEFS, Gomes R. Qual é a sua atitude?: Narrativas de homens jovens universitários sobre os cuidados preventivos com a AIDS. Saude soc [Internet]. 2012[Citado em 29 Abr 2014]; 21(4):916-27. Disponível em: http://www.scielo.br/scielo.php?script=sci _arttext\&pid=S0104-

$12902012000400011 \& \operatorname{lng}=$ pt\&nrm=iso\&tl ng=en

14. Silva FC, Vitalle MSS, Maranhão HS, Canuto MHA, Pires MMS, Fisberg M. Regional differences in knowledge, attitudes, and practice in emergency contraceptive use among health sciences university students in Brazil. Cad saúde pública [Internet]. 2010 [Cited 2014 Abr 20]; 26(9):1821-31. Available from:http://www.scielo.br/pdf/csp/v26n9/1 5.pdf

15. Almeida AS, Nogueira JA, Goldfarb MPL, Batista FL, Barreto AJQ, Moreira MASP, et al. Concepções de jovens sobre o HIV/AIDS e o uso de preservativos nas relações sexuais. Rev Gaúcha Enfermagem [Internet]. 2014 mar [Citado em $22 \mathrm{Abr}$ 2016]; 35(1): 39-46. Disponível em: http://seer.ufrgs.br/index.php/RevistaGauc hadeEnfermagem/article/view/37074/2890 8

16. Rossi-Barbosa, LAR; Ferreira, RC; Sampaio, CA; Guimarães, PN. "Ele é igual aos outros pacientes": percepções dos acadêmicos de Odontologia na clínica de HIV/Aids. Interface (Botucatu) [online]. 2014, vol.18, n.50, pp.585-596. Epub May 09, 2014. ISSN 1807-5762. http://dx.doi.org/10.1590/1807-

57622013.0160 .

Recebido em: 10/12/2015

Aprovado em: 01/07/2016

Publicado em: 31/07/2016 